\title{
Réseaux de cohérence et de cohésion dans la pratique de l'anglais scientifique
}

\section{Anne Magnet}

\section{CpenEdition}

\section{Journals}

Édition électronique

URL : http://journals.openedition.org/asp/2331

DOI : 10.4000/asp.2331

ISBN : 978-2-8218-0380-0

ISSN : 2108-6354

\section{Éditeur}

Groupe d'étude et de recherche en anglais de spécialité

Édition imprimée

Date de publication : 1 décembre 1999

Pagination : 85-98

ISSN : 1246-8185

\section{Référence électronique}

Anne Magnet, "Réseaux de cohérence et de cohésion dans la pratique de l'anglais scientifique », ASp [En ligne], 23-26| 1999, mis en ligne le 15 mars 2011, consulté le 02 mai 2019. URL : http:// journals.openedition.org/asp/2331 ; DOI : 10.4000/asp.2331

Ce document a été généré automatiquement le 2 mai 2019

Tous droits réservés 


\title{
Réseaux de cohérence et de cohésion dans la pratique de l'anglais scientifique
}

\author{
Anne Magnet
}

1 Les enseignants de langue de spécialité travaillent dans des structures qui dispensent des connaissances. Inconsciemment ou consciemment, par ce métier nous vivons le modèle d'organisation du savoir, et donc des structures qui dispensent ce savoir, hérité du Tableau synoptique des connaissances positives (Comte 1928). Depuis 50 ans la classification institutionnelle des disciplines s'est localement transformée, sans que changent sensiblement les références idéologiques, au moins en apparence. C'est pourquoi il paraît intéressant de réfléchir à la manière dont l'organisation de ces structures influence nos représentations mentales des différentes disciplines, et notre place par rapport à ces disciplines. Cette réflexion conduit alors à envisager comment le réseau, réponse transversale à une organisation verticale, permet de trouver une place pour l'enseignant de langue de spécialité.

2 La structure verticale de l'organisation des connaissances est source de rigidité, voire $\mathrm{d}$ 'inhibition dans le fonctionnement de nombreuses disciplines, et en particulier celle que nous pratiquons, l'anglais de spécialité. Comment l'utilisation du concept de réseau(x), conjugué sur différents modes, offre-t-elle une perspective plus ouverte, plus souple, tant dans son aspect humain, sociologique, que dans son aspect cognitif? Comment ce concept peut-il concourir à relier recherche et enseignement ? Je m'appuierai sur l'exemple que je connais le mieux, celui d'un parcours de recherche et d'enseignement de l'anglais de la biologie.

\section{Historique de l'organisation des structures dispensatrices de connaissances}

3 Comme chacun peut le constater dans sa propre université, le modèle positiviste et réaliste est résistant. Rappelons que le positivisme considère que l'humanité passe par 
trois étapes: théologique, métaphysique et positive. Dans l'État Positif, l'esprit humain trouve l'explication ultime des phénomènes en élaborant les lois de leur enchaînement. Pour A. Comte, "le mot positif désigne le réel». Son fameux Tableau synoptique des connaissances positives (1907) était essentiellement une organisation verticale des sciences, avec à la base la mathématique, que Comte définit comme " point de départ de la série »:

Les phénomènes géométriques et mécaniques sont, de tous, les plus généraux, les plus simples, les plus abstraits, les plus irréductibles, et les plus indépendants de tous les autres, dont ils sont au contraire la base. [...] C'est donc la science mathématique qui doit constituer le véritable point de départ de toute éducation scientifique rationnelle, soit générale, soit spéciale, ce qui explique l'usage universel qui s'est établi depuis longtemps à ce sujet, d'une manière empirique, quoiqu'il n'ait eu primitivement d'autre cause que la plus grande ancienneté relative de la science mathématique. (Comte $1907: 2^{\mathrm{e}}$ livre, I, 62-63)

Ceci aboutit à une hiérarchie de valeurs pour la structuration des connaissances où l'on trouve la base occupée par les sciences dures, un sous-ensemble désigné comme sciences molles par lesquelles il faut entendre les autres disciplines scientifiques et enfin la "physiologie sociale ", appellation que Comte donne aux sciences humaines.

Pour organiser ensuite le reste des connaissances, Comte opposait l'ordre dogmatique à

l'ordre historique :

Toute science peut être exposée suivant deux marches essentiellement distinctes, dont tout autre mode d'exposition ne saurait être qu'une combinaison, la marche historique et la marche dogmatique. [...] Le mode dogmatique suppose que tous les travaux particuliers ont été refondus en un système général, pour être présentés suivant un ordre logique plus naturel, qui n'est applicable qu'à une science déjà parvenue à un assez haut degré de développement. À mesure que la science fait des progrès, l'ordre historique d'exposition devient de plus en plus impraticable, tandis que l'ordre dogmatique devient de plus en plus possible, en même temps que nécessaire. (Ibid. : 42-45)

[...] En résultat définitif, la mathématique, l'astronomie, la physique, la chimie, la physiologie et la physique sociale ; telle est la formule encyclopédique qui, parmi le très grand nombre de classifications que comportent les six sciences fondamentales, est seule logiquement conforme à la hiérarchie naturelle et invariable des phénomènes. (Ibid. : 63)

Il est à peu près certain que la force institutionnelle des épistémologies positivistes et réalistes (je rappelle que l'épistémologie est la partie de la philosophie qui étudie l'histoire, les méthodes et les principes des sciences) tient probablement plus à la robustesse de la classification des disciplines scientifiques qu'elles préconisent avec constance, depuis plus d'un siècle, qu'à la qualité de l'adhésion aux hypothèses sur lesquelles elles se fondent.

7 Les critiques nombreuses et virulentes adressées à ce modèle institutionnalisé de l'organisation de la connaissance soulignent, en particulier, son incapacité manifeste à s'accommoder des multiples demandes de connaissances interdisciplinairesformulées par les différents acteurs qui perçoivent la complexité de leurs actions.

8 Le renversement de perspective qui a été initié dans les années 1960 en France, dans le sillage des épistémologies constructivistes a une conséquence inacceptable pour les tenants du paradigme positiviste-réaliste: il ne leur permet plus de se référer en permanence à la « norme du vrai ». La conséquence directe est donc l'abandon du critère de vérité objective, présumé garant de l'enseignabilité des connaissances qu'ils produisent. 
La conceptualisation épistémologique des sciences, argumentée par J. Piaget (1967), H.A. Simon (1969) ou E. Morin (1977), s'appuie sur un mode de connaissance projective, en opposition au mode de connaissance objective. En effet, rien n'oblige à définir une connaissance ou une discipline scientifique exclusivement par son objet (tenu pour indépendant du système observant). Elle peut être définie par son projet, en entendant ce caractère de la connaissance comme le projet cognitif du sujet, qui a conscience d'attribuer au phénomène modélisé une part de ses propres projections.

Au modèle linéaire et hiérarchisé de Comte, Piaget substitue le modèle cyclique de l'organisation de connaissances qui s'irriguent mutuellement au fil d'une boucle circulaire, reliant les sciences logico-mathématiques, aux sciences physiques, puis aux sciences biologiques puis aux sciences psychosociologiques, puis à nouveau cycliquement aux sciences mathématiques. Cette conception permet de casser le modèle linéaire qui hiérarchise les connaissances entre des disciplines nobles (mathématiques et physique) et des disciplines ancillaires (sciences de l'homme et de la société). La conception des disciplines scientifiques par leur projet plutôt que par leur objet a suscité au cours des années 70-80, une sorte de renouvellement de la définition de nombre de disciplines. S'appuyant sur le paradigme des sciences de l'artificiel, et sur l'affirmation de Simon selon laquelle "modéliser est aussi logique que raisonner", sont apparues des sciences comme celles de la cognition. Le concept de science of design ou «science de la conception " chez Simon a également permis d'intégrer de nombreuses disciplines qui n'avaient pas jusqu'alors droit de cité, telles que l'architecture, l'agronomie ou les sciences de l'ingénierie, alors que les épistémologies positivistes ne voyaient par exemple en l'ingénieur qu'un applicateur de connaissances physiques et non un concepteur de projets constructibles.

11 Du Paradigme des sciences de la complexité (Morin 1977) il faut retenir l'idée qu'en réincorporant le sujet dans la connaissance, se trouve construite la boucle récursive du sujet concepteur et du sujet conçu, se connaissant, construisant la connaissance. Il s'agit donc d'une organisation de la connaissance active et perçue dans sa complexité.

En 1971, le biologiste $P$. Weiss modélise et généralise le système cyclique des sciences à travers la métaphore de l'archipel scientifique, et par celle de l'organisation de « lignes maritimes » à travers l'archipel, lui-même inspiré par le modèle de l'île volcanique de Piaget.

Depuis le schéma vertical, fermé et hiérarchisé de Comte, on voit bien le chemin parcouru. La métaphore de l'île volcanique offre un modèle d'interaction complexe et multiforme, puisqu'elle peut à la fois s'élever et s'agrandir, irrégulièrement sous un double effet : celui des disciplines qui agrandissent leur domaine de connaissances en le gagnant sur la mer de «l'empirie» ou qui, s'associant avec leurs voisines, lancent un nouveau cap qui devient peu à peu presqu'île, base ultérieure de nouvelles extensions dites interdisciplinaires. Il faut ajouter à cela le bouillonnement interne du cratère de l'Épistémè qui connaît parfois des explosions internes, lesquelles font remonter à la surface des laves formées autrefois, qui se transforment sous la pression qu'elles ont subie et sous l'action de nouvelles hypothèses.

La question qui reste posée est cependant celle de la généralisation de cette remise en cause. N'y a-t-il pas une majorité silencieuse qui continue de vivre l'épistémologie institutionnelle comme la seule réponse acceptable à la question du statut de la connaissance? Les institutions universitaires ne semblent pas attacher une grande 
importance à ces interpellations, ou pire, quand elles les assimilent dans leur discours, elles n'adaptent pas visiblement leur action pour les prendre en compte. Rien n'est fait au sein des institutions pour que le scientifique s'interroge sur ce que l'on entend par la notion deconnaissance et la constitution de connaissance. L'étonnante inculture épistémologique des chercheurs scientifiques explique qu'une majorité d'entre eux ne s'interrogent pas sur la légitimité des connaissances auxquelles ils font appel pour élaborer, en raisonnant, leurs comportements cognitifs et sociaux.

Les trois grandes questions placées en introduction de Logique et connaissance scientifique (Piaget $1967: 6$ ) sont donc :

- Qu'est-ce que la connaissance ? (la question gnoséologique, sur le fondement de la connaissance)

- Comment est-elle constituée ou engendrée ? (la question méthodologique)

- Comment apprécier sa valeur ou sa validité ? (la question éthique)

Ces questions épistémologiques peuvent paraître éloignées de notre activité de linguistes de spécialité, mais elles conditionnent en réalité notre activité. La suprématie économique américaine, en imposant l'anglais comme langue de communication, entre autres parmi les scientifiques, n'a en fait que susciter des besoins. Ces besoins ne légitiment pas du même coup, nous le savons bien, notre existence.

C'est davantage en nous appuyant sur l'établissement d'un fonctionnement dynamique de l'organisation des connaissances que nous pouvons asseoir notre activité de linguistes de spécialité. Nous représentons l'une des presqu'îles qui ont vu le jour et pour développer notre activité de recherche et d'enseignement nous sommes conduits à suivre des voies multiples sur l'archipel. D'où la nécessité de construire nos propres réseaux ou de nous intégrer à des réseaux existants.

Mon propos est donc de présenter les différents réseaux praticables dans la recherche et l'enseignement de l'anglais scientifique, sachant que la liste est extensible.

\section{Pour une définition fonctionnelle du réseau}

Sans s'arrêter sur le sens étymologique du mot réseau, qui désignait à partir du XII ${ }^{\mathrm{e}}$ siècle, le petit filet qui servait à capturer certains animaux, ou rets, il est intéressant de retenir l'idée de la construction d'un ensemble constitué de mailles, plus ou moins grosses, élaborées avec l'objectif de correspondre à la taille de l'animal visé, c'est-à-dire qu'il y a adéquation entre le projet et les moyens que l'on se donne.

L'anglais dispose de deux mots pour exprimer le réseau : net (qui a évolué en network) et web (qui a une racine commune avec weave), tous deux originaires du vieux frison et $\mathrm{du}$ vieux saxon. Le français ne dispose que d'un mot dont l'étymologie renvoie à l'image du filet, même si celle-ci n'est plus présente dans le terme moderne de réseau, alors que l'anglais offre deux représentations, celle du filet ou celle de la toile d'araignée, d'où d'ailleurs le problème de traduction du mot web de l'informatique par le mot toile, qui n'a pas le même pouvoir évocateur que le terme anglais.

21 Par analogie, le mot réseau a ensuite désigné tout ensemble permanent ou accidentel de lignes, de bandes, etc., entrelacées, entrecroisées, ramifiées plus ou moins régulièrement. 
22 Dans Le réseau et l'infini (1991), P. Boulanger et G. Perelman décrivent les différentes formes de réseaux adaptables à toute activité humaine et les distinguent en fonction de leurs objectifs. Ils donnent une typologie des réseaux en sept groupes :

- les réseaux de proximité, pour se rassembler sur un territoire ou autour d'une idée,

- les réseaux de pression, qui ont pour but de faire aboutir un projet,

- les réseaux de représentation, pour faire connaître une activité en dehors du groupe,

- les réseaux de services pour créer et gérer à plusieurs,

- les réseaux d'influence pour échanger de l'information,

- les réseaux techniques pour mutualiser les moyens,

- les réseaux personnels qui sont activés en fonction des intérêts et des enjeux d'une situation.

Cette typologie générale des réseaux ne peut s'appliquer littéralement à notre activité, mais retenons l'idée que le réseau se définit mieux par sa visée, que par son objet et que le développement $\mathrm{du}$ fonctionnement en réseaux bouscule l'ordre établi : « Le pouvoir n'est plus à celui qui détient l'information mais à celui qui la transmet». (Boulanger \& Perelman 1991)

Un réseau qui se constitue a pour caractéristiques :

- de relier des éléments d'une organisation qui sont au départ isolés en différents points, sans souci préalable de régularité, de symétrie ou de hiérarchie.

- Il est fondé sur l'idée de projet, pour rejoindre l'épistémologie constructiviste, plutôt que sur l'objet.

- Il peut être permanent ou temporaire.

- Il est extensible et se prête à toutes les déformations considérées comme nécessaires à un moment donné.

- Il peut être construit ex nihilo, ou au contraire utiliser des maillons ou des structures existantes.

- Il représente un potentiel de diversité par l'adaptabilité.

- Il se construit dans la transversalité de l'action.

- De par son organisation il possède une capacité d'innovation.

- Il est fondé sur la coopération volontaire et la mise en œuvre de principes de réciprocité.

- Il peut être officiel ou clandestin.

Ce sont ces différentes caractéristiques qui rendent l'utilisation du travail en réseau attrayante.

\section{Les réseaux à activer autour d'une langue de spécialité}

\subsection{Le réseau sociologique}

Être enseignant en langue de spécialité signifie travailler dans une UFR dont la très grande majorité des acteurs sont des spécialistes de disciplines autres que les langues. Dans mon cas ce sont des biologistes. Il est certainement possible de tisser sa propre toile en se satisfaisant d'une situation, à mon avis peu confortable, de simple cohabitation. Je crois que c'est se priver d'un enrichissement très profitable tant pour la recherche que pour l'enseignement.

Construire un réseau sociologique dans son UFR de rattachement consiste à faire état de ses compétences, de ses limites, mais surtout de sa curiosité et de son désir de 
connaissances auprès des collègues spécialistes. Les scientifiques ont forcément leurs travers et leurs défauts, mais jamais je n'ai rencontré chez eux un refus de fournir explications et informations sur leur domaine de spécialité. De plus, il arrive forcément un jour où certains de ces spécialistes ont besoin de l'aide d'un linguiste, en particulier pour les corrections d'articles scientifiques. Cet échange offre le prétexte au démarrage de la construction du réseau. Vous pouvez alors devenir leur informateur dans votre domaine, comme ils deviennent le vôtre dans le leur. Il faut savoir écarter ceux qui ne se posent d'emblée qu'en consommateurs, qui ne comprennent pas l'intérêt de ce travail en équipe, et qui gardent bien ancré en eux le schéma positiviste de l'organisation des connaissances, en étant convaincus d'appartenir au noyau dur. Cela suppose que les contacts avec les spécialistes s'établissent sur différents registres, et que l'enseignant de langue de spécialité fasse au départ un gros effort de compréhension de la structure dans laquelle il travaille, montre un intérêt pour l'ensemble des matières enseignées aux étudiants, c'est-à-dire puisse au moins se faire une idée générale de ce que recouvre chaque unité d'enseignement.

Le réseau a d'ailleurs intérêt à s'étendre aux étudiants. Par exemple, je demande au bout de quelques semaines à mes étudiants de chaque niveau d'apporter en cours la plaquette descriptive de leurs enseignements pour l'année, et de me résumer en anglais en quoi consiste chaque unité. Après quelques années de pratique et en dépit des réformes et des changements de programmes, il est possible, et à mon sens indispensable, d'avoir pour chaque cursus une bonne idée générale du contenu des différents enseignements, afin que les étudiants apprennent à se familiariser avec le vocabulaire adéquat en anglais. Pour certains niveaux comme les licences ou les maîtrises, il est également possible de se procurer les plaquettes d'universités britanniques avec lesquelles l'université à laquelle on appartient a des échanges de type Erasmus ou Socrates. Ceci peut fournir le point de départ d'une comparaison, puis d'un échange sur les différences d'organisation et de programme.

29 Le réseau sociologique que constituent surtout les enseignants-chercheurs, mais aussi les étudiants, permet donc à l'enseignant de langue de spécialité d'acquérir une culture du domaine dans lequel il travaille, ce qui semble une nécessité et de plus facilite grandement son intégration.

\subsection{Le réseau de recherche transversale en anglais de spécialité}

30 Un autre réseau, qui reste souvent à constituer dans de nombreuses universités, est celui qui regrouperait la recherche des différents linguistes de spécialité, selon un axe transversal. Nous connaissons tous à des degrés divers, les réticences de certains anglicistes des voies classiques à l'égard de notre domaine de recherche. Étant donné le développement incontestable du secteur LANSAD ${ }^{1}$, la recherche dans les différents domaines que représente le GERAS ${ }^{2}$ aurait sans doute intérêt à construire son réseau, d'une UFR à l'autre, au sein d'une même université et entre universités. C'est ce qui a commencé à se faire, avec la constitution de sous-groupes de spécialité au sein du GERAS.

\subsection{Le réseau informatique des banques de données}

31 Quand un chercheur en langue de spécialité souhaite effectuer une analyse linguistique à partir d'un corpus de textes, se pose alors le problème de la sélection des textes. En ce qui 
concerne l'anglais scientifique, les chercheurs disposent d'outils qui ont été conçus pour les scientifiques, mais que les linguistes ont tout intérêt à détourner pour la constitution de leur corpus de recherche. Ceci est possible, que l'on travaille en synchronie ou diachronie. En biologie trois banques principales de données sont utilisables. Il s'agit de Biosis, Pascal et Medline. Ces banques de données sont accessibles dans les bibliothèques universitaires de Science ou de Médecine et fournissent tous les résumés d'articles scientifiques parus depuis 1971. Une bibliothécaire sera toujours disponible pour aider les néophytes qui le souhaitent, en particulier pour entrer les mots-clés et les facteurs de limitation ou d'exclusion que l'on souhaite introduire. Ainsi, il est possible de sélectionner les articles scientifiques, qui traitent d'un thème, sur une période donnée, et par exemple provenant uniquement de laboratoires de recherche situés dans un seul pays, ou de plusieurs, que l'on peut définir. La banque fournit le titre, les auteurs, le laboratoire d'origine, les mots-clés, un résumé de l'article et indique s'il s'agit d'un article de recherche ou de vulgarisation. Un premier tri peut donc être effectué avant qu'on demande les versions papier des articles, ou qu'on aille les récupérer sur le Web, si l'on souhaite numériser son corpus. Il est ensuite possible et même nécessaire de faire appel au réseau des spécialistes du domaine pour constituer un corpus représentatif de la discipline, en fonction des critères choisis en commun.

Sans ce recours aux banques de données, et au classement par les spécialistes du domaine, la constitution d'un corpus est aléatoire. Cela a souvent conduit les linguistes à regrouper des articles de spécialités scientifiques qui n'ont pas grand-chose en commun, à composer des corpus très hétéroclites, dont il est difficile de tirer des résultats probants. J'ai constitué mon corpus de recherche avec la méthode ici préconisée, en croisant les informations fournies par les trois banques de données qui ne sont pas équivalentes, en établissant des filtres successifs. Je suis ainsi passée d'une série de 2418 articles fournis par les banques de données sur un thème, à un corpus gérable de 75 textes, jugés les plus importants sur le thème.

Ayant construit mon corpus pour la recherche, je pensais qu'il pouvait fournir une source pour d'autres recherches ou d'autres activités. L'occasion m'en a été fournie par le travail que l'on m'a demandé de faire avec des étudiants de maîtrise de biologie cellulaire spécialisés en nutrition, que je devais préparer à un travail dit de T.E.R. (travail d'étude et de recherche). Pour cette activité, chaque étudiant reçoit une série d'environ dix articles scientifiques en anglais, sur un même thème, dont il doit faire une synthèse écrite en français et une synthèse orale en anglais. Chaque étudiant reçoit son propre lot d'articles. Ce travail personnel de lecture, compréhension, rédaction et expression orale a été intégré à la maquette des maîtrises de biologie il y a deux ans pour pallier le manque d'entraînement à ces savoir-faire chez ces étudiants.

\subsection{Le réseau cognitif du thème de recherche}

Pour l'enseignement au niveau de la maîtrise, le choix de textes scientifiques, différents par le domaine et les thèmes, représente souvent une grande difficulté pour l'enseignant de langue de spécialité, du fait de l'importance du macrotexte propre à chaque article. De plus, dans mon expérience préalable, cela m'a souvent conduite à un sentiment d'éparpillement. Comme notre rôle ne peut se réduire à l'acquisition d'un lexique spécialisé, et qu'il serait vain de chercher à couvrir toutes les spécialités enseignées aux étudiants, j'ai choisi cette année de me servir du corpus constitué pour ma thèse et donc 
de centrer tout le cours autour d'un seul thème biochimique : les acides gras essentiels et d'exploiter ce thème, à partir des recherches effectuées par différents spécialistes. Ce thème est particulier car il offre un terrain d'études à différents spécialistes de biologie. Le thème participe donc d'un réseau cognitif dans lequel coopèrent les biochimistes qui mettent le caractère essentiel de ces acides gras en évidence, les nutritionnistes spécialistes des lipides, qui étudient le rôle de ces acides gras dans l'apparition ou le développement de nombreuses pathologies: hypertension, diabète, maladies cardiovasculaires, les immunologistes qui cherchent à comprendre le rôle joué par les acides gras essentiels dans la défense immunitaire, les spécialistes de neuro-science, qui analysent les déficits neurologiques chez les sujets privés de ces acides gras particuliers. Tous ces spécialistes partagent un projet commun: construire le macrotexte sur les acides gras essentiels. Cet ensemble de connaissances peut donc fonctionner comme un réseau ouvert, dans lequel il est possible de puiser, en y trouvant une cohérence, tant pour la recherche que pour l'enseignement.

\subsection{La diachronie comme réseau de cohésion}

Il est généralement admis en recherche sur l'anglais scientifique comme dans la pratique de l'enseignement de la langue de spécialité, que plus les textes choisis sont récents, mieux cela vaut. Ceci est sans doute dû au développement de la communication dans notre société et au souci d'être toujours en prise directe avec l'actualité, en science comme ailleurs. Cette approche n'est pas à remettre en question, car nous savons qu'elle peut être un des atouts des langues de spécialité. Pourquoi nous priverions nous de l'attrait du neuf, en particulier dans l'enseignement en premier cycle ? Cependant, il peut être utile et intéressant, en tout cas dans le domaine scientifique, de travailler quelquefois avec une approche diachronique, car cela permet d'envisager l'ensemble des facteurs du réseau de la publication scientifique. J. Martin rappelait au séminaire de Montpellier de 1996 que : "le sens est produit en interférence entre les objets et les sujets, et qu'il faut envisager l'ensemble des facteurs ». En m'appuyant sur l'épistémologie constructiviste rapportée au début de cette communication, qui privilégie le projet par rapport à l'objet, je dirai qu'en sélectionnant un seul objet de recherche, mais en l'analysant avec le recul du temps, il est alors possible de se concentrer sur le projet dans ses différents aspects (construction du fait scientifique, processus développés pour y parvenir, stratégies de validation des résultats).

L'organisation d'un thème de recherche et d'enseignement en réseau diachronique rend la recherche plus ciblée et offre, en ce qui concerne les étudiants, l'occasion de réfléchir sur le fonctionnement de la Science et la genèse des connaissances.

Pour ma recherche, j'étudie la construction du fait scientifique à travers une analyse discursive et linguistique des articles scientifiques, sur une période de 70 ans, sur un seul thème scientifique. Le thème n'a pas été aisé à définir car il fallait trouver un thème présent en 1929 dans la recherche en biochimie de la nutrition et toujours actuel. La nutrition est elle même un domaine en pleine expansion. Certaines données, telles que l'étude d'un journaliste scientifique du Nouvel Observateur indiquent que

60000 nouvelles études scientifiques ont été publiées en 1997 dans le domaine de la nutrition, faisant de cette discipline la toute première des sciences de la vie, devant la génétique. (Souccar $1998: 3$ ) 
Représenter l'article scientifique comme l'acte plus ou moins isolé d'une équipe de ensuite à la communauté de spécialistes, puis à la société en général, constitue la position des positivistes-réalistes. Si, au lieu de cela, on se représente l'activité scientifique comme une activité humaine de construction à partir du réel et non de découverte, on aboutit dans chaque spécialité à la construction de ce que Jacky Martin a nommé le macrotexte, c'est-à-dire l'ensemble de ce qui est supposé connu par les lecteurs de l'article, et qui conditionne la compréhension et la production du discours spécialisé. En choisissant de travailler en diachronie, avec des articles portant sur un seul thème, il est possible de rendre la structure dynamique et métastable du discours scientifique (le terme « métastable » définit un système qui n'est pas stable en théorie, mais qui paraît tel, en raison d'une vitesse de transformation très faible).

Plus que le lexique spécialisé ou les caractéristiques linguistiques de surface, ce sont les stratégies développées par les auteurs pour avoir le droit de contribuer au paradigme de la spécialité, qui fondent la cohérence et la cohésion du discours scientifique. Il faut entendre par cohérence l'absence de contradiction entre deux discours. Or il y a, dans la construction du discours scientifique des périodes de crise et de contradiction, mais la résolution des crises se fait toujours par l'abandon d'un paradigme au profit d'un autre (Kuhn 1962). C'est à ce prix que se fait la construction du macrotexte, et que sa cohérence est assurée. La cohésion (du latin cohedere : adhérer ensemble) du discours scientifique est assurée par le fait que, pour qu'un texte s'inscrive dans le macrotexte, pour qu'une partie adhère à l'ensemble, il faut des outils de liaison (de "suture " selon J. Martin) et des activateurs de mémoire. Un texte n'est pas plus une suite de mots qu'un article scientifique n'est une production textuelle isolée. Le texte produit par les scientifiques est à concevoir comme un tissu (du latin textus dont l'origine est le verbe textere: tisser). "Speaking or writing is weaving ", comme le dit W. Rotgé (1998 : 183). Ceci nous ramène à l'étymologie du terme anglais web, fourni précédemment, comme l'une des possibles traductions du mot réseau. 

d'interférences d'une part entre les objets et les sujets, entre le sujet et la communauté de spécialistes et, d'autre part, mais ceci n'est pas notre propos ici, entre le réseau de spécialistes et la société en général. Il s'agit du réseau de dissémination des connaissances.

\subsection{La pédagogie comme un réseau d'échange cognitif}

J'ai indiqué que l'objectif de la formation en langue de spécialité des étudiants de maîtrise était de leur offrir une préparation à leur T.E.R. (travail d'étude et de recherche) dans leur domaine de spécialité.

- À partir du corpus de recherche, j'ai sélectionné douze articles qui représentaient des étapes importantes dans l'élaboration du macrotexte sur les acides gras essentiels.

- Chaque article a été confié à un binôme d'étudiants pour la présentation d'une synthèse orale.

- Les présentations orales suivent l'ordre chronologique des publications.

- Chaque présentation orale comporte en introduction l'activation du macrotexte ou : « What the reader needs to know before starting to read the article ». Ceci peut paraitre un exercice difficile mais il faut se souvenir qu'un article scientifique comporte une très grande partie de déjà dit, peut-être 80 \%, d'après l'estimation de J. Martin. Pour les premiers articles, le macrotexte est relativement léger.

- Le binôme présente l'article devant le groupe d'étudiants qui ne l'a pas lu. Il est demandé aux étudiants rapporteurs de préparer des transparents (dont le contenu scientifique aura été vérifié par un spécialiste de la discipline), qui ont deux fonctions : soutien à la compréhension pendant la présentation par la représentation graphique, iconographique, symbolique des informations et aide à la restitution de l'information essentielle par le groupe après la présentation de l'article par le binôme.

- Le groupe prend des notes et pose des questions sur ce qui n'a pas été compris, ou en cas d'incertitude.

- L'information importante est restituée oralement par le groupe, avec l'aide de notes, de transparents (ou en dernier recours à l'aide de questions préparées par l'enseignant, si les étudiants éprouvent trop de difficultés).

À partir du deuxième exposé, une partie du macrotexte est connue, ce qui élimine certaines difficultés de prononciation du lexique spécialisé.

- Comme le grand thème est connu à l'avance, les étudiants faibles ont moins de difficultés de compréhension. En revanche, le travail d'expression peut se concentrer sur l'utilisation du lexique et de la syntaxe d'orientation scientifique (cf. Minimum Competence in Scientific English , Upjohn et al., 1991). Le linguiste doit faire l'effort d'acquérir certains concepts scientifiques propres au thème, pour lesquels il pourra se faire aider par les spécialistes du domaine. L'effort est limité puisqu'il ne porte que sur un thème. L'explicitation du macrotexte par les étudiants lui permet à chaque fois de réactiver ses connaissances, d'en discuter, de demander des suppléments d'information aux spécialistes ou aux étudiants, pour qui cela constitue un excellent travail d'expression orale.

- Une fois le travail oral accompli, chaque binôme reprend son article pour en étudier quelques caractéristiques linguistiques et discursives (verbes, mots de liaison, construction des parties IMRAD, etc.) Ce travail est mis en commun, exploité par l'enseignant qui peut en tirer des conclusions retransmises au groupe. 


\section{Conclusion}

Deux questions ont donc été soulevées ici, qui concernent, chacune à des degrés divers, les enseignants de langue de spécialité: d'une part leur positionnement au sein de l'organisation des structures dispensatrices de connaissances qu'est l'Université. D'autre part la possibilité de lier recherche et enseignement dans une approche non généralisable, mais exploitable avec certains étudiants de deuxième cycle pour qui il est souhaitable de travailler autour de documents authentiques.

Le sentiment de pratiquer une discipline ancillaire est un sentiment répandu chez les linguistes de spécialité. Si nous ne souhaitons pas nous satisfaire de la place que nous faisait l'épistémologie positiviste-réaliste, et si nous souhaitons répondre à la forte demande exprimée par les scientifiques et les étudiants en science, nous avons tout intérêt à multiplier notre capacité à échafauder des réseaux fonctionnels humains, cognitifs, permanents ou temporaires, pour répondre à leurs attentes. Depuis les années 70 , les universités ont augmenté le nombre des disciplines reconnues et enseignées en leur sein. Le fossé entre disciplines fondamentales et appliquées s'est réduit, les frontières entre les disciplines s'estompent. Il y a fort à parier que les nouvelles voies se feront essentiellement à partir des chemins de traverse. L'interdisciplinarité fait émerger certaines voies nouvelles de recherche. C'est sur cet axe que se situent les langues de spécialité. Changer les structures institutionnelles dans lesquelles nous travaillons s'avère une tâche très lourde, et très longue qui nous est peu accessible. Organiser des réseaux fonctionnels permet de faire la preuve d'un savoir-faire, fondé sur des réalités de terrain. Par la souplesse d'organisation et les moyens plus limités qu'il suppose, le travail en réseau appliqué aux domaines sociologique, cognitif, technologique, ainsi qu'à celui de la recherche pratiquée sur des axes transversaux, est une réponse aux rigidités de l'épistémologie institutionnelle. Enfin, chacun a pu remarquer combien le fait de lier recherche et enseignement peut participer à la reconnaissance de l'enseignant de spécialité au sein de son UFR.

\section{BIBLIOGRAPHIE}

Boulanger, Pierre \& Guy Perelman. 1991. Le réseau et l'infini. Paris : Nathan.

Chalmers, Alan. F. 1976. What is this Thing Called Science? An Assessment of the Nature and Status of Science and its Methods. St Lucia, Australie : University of Queensland Press.

Comte, Auguste. 1907. Cours de philosophie positive. Paris : Éditions Schleicher Frères.

Gunnarson, Britt-Louise. 1989. « LSP texts in a diachronic perspective ». In Lauren, Christen \& Marianne Nordman (dir.), Special Language. Bristol : Taylor \& Francis.

Halliday, Michael A. K. \& James R. Martin. 1993. Writing Science, Literary and Discursive Power. Londres : Falmer Press. 
Kuhn, Thomas. 1983. La structure des révolutions scientifiques. Paris : Flammarion.

Le Moigne, Jean-Louis. 1995. Les épistémologies constructivistes. Paris : Presses Universitaires de France.

Martin, Jacky. 1995. « Convergences et divergences du discours scientifique ». Séminaire de DEA, Université Paul Valéry, Montpellier 3.

Martin, Jacky. 1996a. « Les enjeux du discours scientifique : la stratégie de véridiction ». ASp 11-14, 13-31.

Martin, Jacky. 1996b. «Linguistique du discours scientifique », Cours du DEA de langue de spécialité de Bordeaux 2, Montpellier 3, Paris 4 et Toulouse 1.

Morin, Edgar. 1977. La méthode, t.I : La nature de la nature. Paris : Seuil Points.

Myers, Greg. 1990. Writing Biology, Texts in the Social Construction of Scientific Knowledge. Madison, WI : The University of Wisconsin Press.

Piaget, Jean. 1967. Logique et connaissance scientifique. Paris : Gallimard-Encyclopédie de la Pléiade.

Simon, Harry A. The Sciences of the Artificial. Cambridge, MA : MIT Press, 1981.

Souccar, Thierry. 1998. «La révolution dans les assiettes, la santé à pleines dents ». Le Nouvel Observateur 1761, 3-6.

Rotgé, Wilfrid. 1998. « Le point sur la cohésion en anglais, application à un texte de fiction ». Anglophonia 4, 181-199.

Upjohn, Jonathan, Blattes, Sue \& Véronique Jans. 1991. Minimum Competence in Scientific English. Grenoble : Presses Universitaires de Grenoble.

\section{NOTES}

1. Langues pour Spécialistes d'Autres Disciplines : sigle proposé par Michel Perrin au colloque d'Aix en Provence 1993 sur « L'enseignement des langues aux non-spécialistes ».

2. Groupe d'Étude et de Recherche en Anglais de Spécialité

\section{RÉSUMÉS}

Cet article s'attache à montrer comment la recherche en anglais scientifique à partir de l'étude d'un corpus constitué en diachronie sur un thème scientifique de biologie (les acides gras essentiels) peut fournir une base pour l'enseignement de l'anglais de spécialité à des étudiants de maîtrise. Il est d'abord procédé à un rapide parcours des différentes épistémologies qui se sont succédé pour proposer l'organisation des savoirs scientifiques. Il est ensuite montré comment les enseignants de langues de spécialité sont confrontés consciemment ou inconsciemment au modèle résistant de l'organisation du savoir et donc des structures qui dispensent le savoir, héritées $d u$ positivisme. Une définition du terme réseau est proposée ainsi que les divers registres dans lesquels le concept de réseau peut fournir un mode de fonctionnement: sociologique, cognitif, diachronique, épistémologique et pédagogique. 
This paper investigates how research on scientific English built on the study of a diachronic corpus around a specific biological theme (essential fatty acids) can be used as a basis for a Maitrise training course in ESP. First, the various epistemologic schools are rapidly described to recall the different trends in the conceptual organization of knowledge. We then explain how ESP teachers are consciously or unconsciously confronted with or influenced by the resisting positivist model of knowledge structuring. A definition of the word 'network' is proposed, as well as the various registers in which the concept of network can offer a mode of action for ESP teaching: sociological, cognitive, diachronic, epistemological and pedagogical.

\section{INDEX}

Mots-clés : article de recherche scientifique, diachronie, didactique, épistémologie, linguistique de corpus, réseau de cohérence

Keywords : coherence network, corpus linguistics, diachrony, epistemology, scientific research article, training course

\section{AUTEUR}

\section{ANNE MAGNET}

Anne Magnet est professeur d'anglais à l'UFR des Sciences de la vie de l'Université de Bourgogne. Sa thèse, préparée sous la co-direction des Professeurs M. Ratié, de l'Université de Bourgogne, et J. Martin, de l'Université de Montpellier 3, a pour titre «L'Étude diachronique de l'article de recherche scientifique en anglais dans la sous-discipline 'nutrition' ». Ses travaux de recherche se concentrent sur l'analyse discursive et linguistique du discours scientifique. Anne.Magnet@ubourgogne.fr 